\title{
INJECTIVE WEAK SOLUTIONS IN SECOND-GRADIENT NONLINEAR ELASTICITY
}

\author{
Timothy J. Healey ${ }^{1}$ And Stefan Krömer ${ }^{2}$
}

\begin{abstract}
We consider a class of second-gradient elasticity models for which the internal potential energy is taken as the sum of a convex function of the second gradient of the deformation and a general function of the gradient. However, in consonance with classical nonlinear elasticity, the latter is assumed to grow unboundedly as the determinant of the gradient approaches zero. While the existence of a minimizer is routine, the existence of weak solutions is not, and we focus our efforts on that question here. In particular, we demonstrate that the determinant of the gradient of any admissible deformation with finite energy is strictly positive on the closure of the domain. With this in hand, Gâteaux differentiability of the potential energy at a minimizer is automatic, yielding the existence of a weak solution. We indicate how our results hold for a general class of boundary value problems, including "mixed" boundary conditions. For each of the two possible pure displacement formulations (in second-gradient problems), we show that the resulting deformation is an injective mapping, whenever the imposed placement on the boundary is itself the trace of an injective map.
\end{abstract}

Mathematics Subject Classification. 74B20, 49K20.

Received December 27, 2007.

Published online July 19, 2008.

\section{Introduction}

Existence theorems for properly formulated problems of finite, nonlinear elasticity in 2 or 3 dimensions are somewhat scarce and incomplete. A celebrated result of elastostatics, due to Ball [2], provides for the existence of minimizers of the potential energy in the Sobolev space $W^{1, p}$. From a historical point of view, the construction in [2] is quite delicate - accounting for the lack of convexity of the stored energy function via polyconvexity while enforcing the physical requirement that the energy density "blows up" as the local volume ratio approaches zero. However, as a direct consequence of the latter, it is generally not known if such minimizers correspond to actual weak equilibrium solutions of the Euler-Lagrange equations, $c f$. [4].

The promise of models incorporating higher-gradient "interfacial" energy is that the analysis should be simpler and potentially lead to solutions of the unregularized problem in the limit of vanishing interfacial energy. We focus on the former in this paper; the basic existence of a minimizer is indeed routine. However, if we correctly maintain the physical requirement that the classical energy density function blows up as the local volume ratio

\footnotetext{
Keywords and phrases. Gradient estimate, injective deformations, Euler-Lagrange equation, nonlinear elasticity.

1 Cornell University, Ithaca, NY 14853, USA.

2 Universität Augsburg, 86135 Augsburg, Germany. stefan.kroemer@math.uni-augsburg.de
} 
goes to zero, the question of weak solutions to the corresponding Euler-Lagrange equations is again delicate. It is precisely this question that we pursue in this paper.

The outline of the work is as follows. In Section 2 we formulate the minimization problem for the pure displacement problem on a bounded domain. We take the potential energy density to be the sum of two functions - a convex function of the second gradient and a rather general function of the first gradient. As in classical nonlinear elasticity, we require that the latter function grow unboundedly as the determinant of the gradient approaches zero. We then routinely establish the existence of a minimizer in $W^{2, p} \cap C^{1, \alpha}$, where the latter denotes the usual Hölder space for some $\alpha \in(0,1)$. Section 3 is the heart of the paper. In particular, we demonstrate that the determinant of the first gradient of any admissible deformation with finite energy is strictly positive on the closure of the domain, after which we readily show that the minimizer yields a weak solution of our second-gradient problem. If the prescribed boundary placement is the trace of an injective mapping, we then show that the total deformation is also injective whenever its energy is finite. In Section 4 we indicate how our approach also leads to the existence of weak solutions for other boundary value problems, including "mixed" boundary conditions. Unlike Section 3, this requires modest assumptions on the regularity of the boundary.

Our results are applicable to deformations of finite energy in classical nonlinear elasticity, assuming sufficient smoothness (of class $C^{1, \alpha}$ ). For energy minimizers in this setting, our results overlap with those of [6] ( $c f$. also [5, 13]; the latter presents a sharper result for the case $n=2$ ). We discuss this more fully in our final remarks in Section 5 .

\section{Problem Formulation}

We consider a bounded domain $\Omega \subset \mathbb{R}^{n}, n \geq 2$, and we associate its closure $\bar{\Omega}$ with a reference configuration for a material body. Deformations of the body are orientation-preserving mappings $f: \bar{\Omega} \mapsto \mathbb{R}^{n}$, i.e., $f(x)$ is the placement of the material point occupying position $x$ in the reference configuration. In the sequel we denote the set of all linear transformation of $\mathbb{R}^{n}$ into itself by $L\left(\mathbb{R}^{n}\right)$, and $B L\left(\mathbb{R}^{n}\right)$ denotes the set of all bilinear transformations of $\mathbb{R}^{n} \times \mathbb{R}^{n}$ into $\mathbb{R}^{n}$ that are symmetric in their arguments, i.e., $A[x, y]=A[y, x] \forall x, y \in \mathbb{R}^{n}$, $A \in B L\left(\mathbb{R}^{n}\right)$. For each fixed $x \in \mathbb{R}^{n}$, the gradient and second gradient of the deformation belong to $D f(x) \in$ $L\left(\mathbb{R}^{n}\right)$ and $D^{2} f(x) \in B L\left(\mathbb{R}^{n}\right)$, respectively. The determinant of $F \in L\left(\mathbb{R}^{n}\right)$ is denoted $\operatorname{det} F$, and $G L^{+}\left(\mathbb{R}^{n}\right)$ denotes the set of all $F \in L\left(\mathbb{R}^{n}\right)$ such that $\operatorname{det} F>0 . S O(3) \subset G L^{+}\left(\mathbb{R}^{n}\right)$ is the set of all proper rotations. All function spaces employed in this work contain $n$-vector-valued functions. For convenience and without loss of generality, we consistently shorten the notation, e.g., we write $L^{p}(\Omega)$ instead of $L^{p}\left(\Omega, \mathbb{R}^{n}\right), C^{m, \alpha}(\bar{\Omega})$ instead of $C^{m, \alpha}\left(\bar{\Omega}, \mathbb{R}^{n}\right)$, etc.

The elastic body is presumed homogeneous, and its material response is described by two classes of energy density functions, $\Phi: G L^{+}\left(\mathbb{R}^{n}\right) \rightarrow[0, \infty)$ and $\Psi: B L\left(\mathbb{R}^{n}\right) \rightarrow[0, \infty)$, each of which satisfy the principle of objectivity:

$$
\Psi(Q \circ G)=\Psi(G), \Phi(Q \circ F)=\Phi(F) \quad \forall Q \in S O(3) .
$$

Let $f_{o}: \bar{\Omega} \mapsto \mathbb{R}^{n}$ be given, which specifies the placement of the boundary $\partial \Omega$, and which is presumed to have the following properties:

$$
f_{o} \in W^{2, p}(\Omega) \cap C^{1, \beta}(\bar{\Omega}), \operatorname{det} D f_{o}>0 \text { on } \bar{\Omega},
$$

where $\beta \in(0,1)$ and $p$ are constants satisfying

$$
p>n \text { and } \beta \geq(p-n) / n .
$$

The total potential energy of the elastic body is given by

$$
E(u):=\int_{\Omega}\left[\Psi\left(D^{2} f\right)+\Phi(D f)\right] \mathrm{d} x, \text { where } f=u+f_{0} .
$$


Since $f$ is a deformation, we seek to minimize (2.4) over the admissible class of functions

$$
\mathcal{A}:=\left\{u \in W_{o}^{2, p}(\Omega): \operatorname{det}\left(D u+D f_{o}\right)>0 \text { a.e. in } \Omega\right\} .
$$

We assume the following physically reasonable conditions for the energy density functions:

$$
\begin{gathered}
\Psi: B L\left(\mathbb{R}^{n}\right) \rightarrow[0, \infty) \text { is continuous and convex, } \\
\frac{1}{C}|G|^{p} \leq \Psi(G) \leq C|G|^{p}+C_{1}
\end{gathered}
$$

for all $G \in B L\left(\mathbb{R}^{n}\right)$, and

$$
\begin{gathered}
\Phi: G L^{+}\left(\mathbb{R}^{n}\right) \rightarrow[0, \infty) \text { is continuous, } \\
\Phi(F) \rightarrow+\infty \text { as } \operatorname{det} F \searrow 0 \\
\Phi(F):=+\infty \text { if } \operatorname{det} F \leq 0
\end{gathered}
$$

cf. [2]. Here, $C>0$ and $C_{1} \geq 0$ are constants.

It's worth mentioning that, since the higher-gradient term in (2.4) is convex, we do not require much structure on the stored energy function $\Phi(F)$, e.g., polyconvexity. In particular, our results are applicable to cases in which $\Phi(F)$ has a multi-well structure, appropriate for phase-transition problems. With these assumptions in hand, we obtain a rather standard result:

Proposition 2.1. Given (2.2), (2.3), (2.6) and (2.7), then $E$ attains its minimum on $\mathcal{A}$, i.e., there is at least one $u_{*} \in \mathcal{A}$ such that $E\left(u_{*}\right)=\inf _{u \in \mathcal{A}} E(u)$.

Proof. Let $\left\{u_{j}\right\} \subset \mathcal{A}$ denote a minimizing sequence so that $E\left(u_{j}\right) \rightarrow \inf E$ as $j \rightarrow \infty$. Observe that $E(0)<\infty$, and thus, we may assume that $\left\{E\left(u_{j}\right)\right\}$ is bounded in $\mathbb{R}$. By virtue of (2.6), we deduce that $\int_{\Omega}\left|D^{2} u_{j}+D^{2} f_{o}\right|^{p} \mathrm{~d} x$ is bounded, and, since $f_{o} \in W^{2, p}(\Omega)$ is fixed, $\int_{\Omega}\left|D^{2} u_{j}\right|^{p} \mathrm{~d} x$ is also bounded. By a version of Poincaré's inequality, we conclude that $\left\{u_{j}\right\}$ is bounded in $W_{o}^{2, p}(\Omega)$. Let $u_{*}$ denote the weak limit of the sequence in $W_{o}^{2, p}(\Omega)$. By compact embedding, passing to a subsequence if necessary, we have that $u_{j} \rightarrow u_{*}$ strongly in $W^{1, p}(\Omega)$, with $u_{j}(x) \rightarrow u_{*}(x)$ and $D u_{j}(x) \rightarrow D u_{*}(x)$ for almost every $x \in \Omega$. Fatou's lemma then delivers

$$
\liminf _{j \rightarrow \infty} \int_{\Omega} \Phi\left(D u_{j}+D f_{o}\right) \mathrm{d} x \geq \int_{\Omega} \Phi\left(D u_{*}+D f_{o}\right) \mathrm{d} x .
$$

Now from (2.6), it follows that the functional $\widetilde{E}(w):=\int_{\Omega} \Psi\left(D^{2} w\right) \mathrm{d} x, \widetilde{E}: W^{2, p}(\Omega) \rightarrow \mathbb{R}$ is convex and hence weakly lower semi-continuous, e.g., [10]. In particular, we have

$$
\liminf _{j \rightarrow \infty} \int_{\Omega} \Psi\left(D^{2} u_{j}+D^{2} f_{o}\right) \mathrm{d} x \geq \int_{\Omega} \Psi\left(D^{2} u_{*}+D^{2} f_{o}\right) \mathrm{d} x .
$$

Together, (2.8) and (2.9) imply that $\inf E \geq E\left(u_{*}\right)$, i.e., $u_{*}$ is a global minimizer. Clearly $u_{*} \in \mathcal{A}$. Otherwise we would have $D u_{*}+D f_{o}=0$ a.e. on some subset of $\Omega$ of non-zero measure, which, in view of (2.7), would give $E(0)<E\left(u_{*}\right)=\infty$.

Remark 2.2. The presumed Hölder-continuity of $D f_{o}$ is not necessary for the purposes of Proposition 2.1. However, by (2.2), (2.3) and continuous embedding [1], observe that each $u+f_{o} \in C^{1, \lambda}(\bar{\Omega})$ for $u \in W_{o}^{2, p}(\Omega)$, with $\lambda=(p-n) / p$. Thus the second term in (2.4) is well defined in the absence of a growth condition, which would otherwise be required, at least if the domain is not smooth enough for an embedding theorem to hold for $f_{0}$. We also mention that a version of Proposition 2.1 holds when $1<p \leq n$ if we add an appropriate growth condition on $\Phi$. Our results that follow depend crucially upon (2.2) and (2.3). 


\section{INJECTIVE WEAK SOLUTIONS}

The existence of a minimizer within the setting of Section 2 is much simpler than that of classical nonlinear elasticity $[2,7]$. However, similar to the classical formulation, the physically reasonable growth condition $(2.7)$ precludes any immediate conclusions concerning whether the minimizer is actually a weak solution of the EulerLagrange equations. The difficulty stems from the fact that, due to the construction of a minimizer $u_{*} \in \mathcal{A}$ (cf. (2.5)), the functional $E$ need not be Gâteaux differentiable at $u_{*}$. In this section we demonstrate a way to overcome this difficulty. For that purpose, we first need sharper hypotheses in addition to those laid out in Section 2:

$$
\begin{gathered}
\operatorname{det} D f_{o} \geq \bar{c}>0 \text { on } \bar{\Omega}, \\
\Phi(F) \geq \frac{1}{C}(\operatorname{det} F)^{-q}, \quad \forall F \in G L^{+}\left(\mathbb{R}^{n}\right),
\end{gathered}
$$

where $\bar{c}>0, C>0$ and $q>0$ are constants, with

$$
q \geq \frac{p n}{p-n}
$$

and $p>n$ as before.

Our main result is the following:

Theorem 3.1. Given (2.2), (2.3), (2.6) and (3.1)-(3.3), then for every $\kappa \geq \inf E$ there is a constant $K>0$ such that

$$
\operatorname{det}\left(D u+D f_{o}\right) \geq K \text { on } \bar{\Omega}
$$

holds for every $u \in \mathcal{A}$ with $E(u) \leq \kappa$. Here, $K$ generally depends on $\kappa, f_{o}, n, p, q$, the constants in (2.6), (3.1), (3.2), and the measure of $\Omega$, but not on $u$.

In particular, we obtain:

Corollary 3.2. Given the hypotheses of Proposition 2.1 and Theorem 3.1, then there is a constant $K>0$ such that every minimizer $u_{*}$ satisfies

$$
\operatorname{det}\left(D u_{*}+D f_{o}\right) \geq K \text { on } \bar{\Omega},
$$

where $K$ generally depends on $\inf E, f_{o}, n, p, q$, the constants in (2.6), (3.1), (3.2), and the measure of $\Omega$, but not on $u_{*}$.

Proof. From Proposition 2.1, we have the existence of at least one minimizer $u_{*} \in \mathcal{A}$, i.e., $E\left(u_{*}\right)=\inf E$, and Theorem 3.1 yields the assertion.

If $\Psi$ and $\Phi$ are smooth enough, we immediately conclude:

Corollary 3.3. In the setting of Corollary 3.2, suppose that $\Psi$ and $\Phi$ are of class $C^{1}$, with

$$
|D \Psi(G)| \leq C_{2}\left(|G|^{p-1}+1\right) \quad \forall G \in B L\left(\mathbb{R}^{n}\right),
$$

for some positive constant $C_{2}$. Then any minimizer $u_{*}$ satisfies the weak form of the Euler-Lagrange equation, viz.,

$$
\int_{\Omega}\left[D \Psi\left(D^{2} u_{*}+D^{2} f_{o}\right) \cdot D^{2} \phi+D \Phi\left(D u_{*}+D f_{o}\right) \cdot D \phi\right] \mathrm{d} x=0,
$$

for all test functions $\phi \in W_{o}^{2, p}(\Omega)$.

Proof. By standard arguments [8], the smoothness and growth condition (3.6) ensure that the functional $E$ is Gâteaux differentiable on $\mathcal{A}_{\eta}:=\left\{u \in \mathcal{A}: \operatorname{det}\left(D u+D f_{o}\right) \geq \eta\right.$ on $\left.\bar{\Omega}\right\}$, for each fixed $\eta>0$. Hence (3.7) holds for every $\phi \in C_{o}^{\infty}(\Omega)$; the left side of (3.7) is the Gâteaux derivative of $E$ at $u_{*}$ in the direction of $\phi$. By a density argument, we infer the validity of (3.7) for all $\phi \in W_{o}^{2, p}(\Omega)$. 
Another important ramification of Theorem 3.1 is:

Corollary 3.4. Given the hypotheses of Theorem 3.1, assume that

$$
\begin{gathered}
f_{o} \text { is injective on } \bar{\Omega}, \text { and } \\
\operatorname{int} \bar{\Omega}=\Omega .
\end{gathered}
$$

Then for every $u \in \mathcal{A}$ with finite energy, the mapping

$$
f:=u+f_{o} \text { is injective on } \bar{\Omega} \text {. }
$$

Proof. We first note that $f \in C^{1, \alpha}(\bar{\Omega})$, for $\alpha:=(p-n) / p$, cf. Remark 2.2. Clearly we have $f(x)=f_{o}(x)$ $\forall x \in \partial \Omega$. The assertion then follows from (3.4), (3.8), (3.9) and a well-known argument employing the Brouwer degree, e.g., [7].

The proof of Theorem 3.1 relies upon the following:

Lemma 3.5. Let $J \in C^{\alpha}(\bar{\Omega}), \alpha \in(0,1)$, be such that $J(x)>0$ a.e. in a bounded domain $\Omega \subset \mathbb{R}^{n}$. In addition, suppose $\int_{\Omega}(J(x))^{-q} \mathrm{~d} x \leq L$ for some $q \geq n / \alpha$ and $\|J\|_{C^{\alpha}(\bar{\Omega})} \leq M$, where $L, M$ are positive constants. Then for every $\delta>0$, there is a positive constant $c$ such that

$$
J(y) \geq c \quad \forall y \in \Omega_{\delta}:=\{x \in \Omega: \operatorname{dist}(x ; \partial \Omega) \geq \delta\},
$$

where $c$ depends upon $\delta, M, L, q$ and $n$ but not on $y, J$ or $\Omega$.

Proof. For a given $\delta>0$, define a function $h:[0, \infty) \rightarrow(0, \infty) \cup\{+\infty\}$ via

$$
h(t):=\omega_{n-1} \int_{0}^{\delta}\left(t+M r^{\alpha}\right)^{-q} r^{n-1} \mathrm{~d} r,
$$

where $\omega_{n-1}$ denotes the $(n-1)$-dimensional measure of the unit sphere in $\mathbb{R}^{n}$. We note that $h$ is strictly decreasing with $h(t) \rightarrow \infty$ as $t \searrow 0$, the latter following from the fact that the exponent of " $r$ " in (3.10) (at $t=0)$ is equal to $-\alpha q+n-1 \leq-1$ ( since $q \geq n / \alpha)$. Thus, there is a positive constant $c$ such that

$$
h(t) \leq L \text { if and only if } t \geq c
$$

By assumption

$$
J(x) \leq J(y)+M|x-y|^{\alpha},
$$

for every pair of points $x, y \in \Omega$. By virtue of (3.10) and (3.12), we find, for every fixed $y \in \Omega_{\delta}$,

$$
h(J(y)) \leq \int_{B_{\delta}(y)}(J(x))^{-q} \mathrm{~d} x \leq L .
$$

The result now follows directly from (3.11) and (3.13).

Remark 3.6. If $J$ only belongs to $C_{l o c}^{\alpha}(\Omega)$, the proof above still goes through with minor modifications. We then obtain that $J \geq c>0$ in $\Omega_{2 \delta}$. Here, the constant $M$ has to be replaced by $M_{\delta} \geq\|J\|_{C^{\alpha}\left(\Omega_{\delta}\right)}$.

Proof of Theorem 3.1. For any $u \in \mathcal{A}$, we have that

$$
J:=\operatorname{det}\left(D u+D f_{o}\right)>0 \text { a.e. in } \Omega \text {. }
$$


As observed before ( $c f$. Rem. 2.2), note that bounded energy implies that $u+f_{o}$ is bounded in $C^{1, \alpha}(\bar{\Omega})$, for $\alpha:=(p-n) / p$, i.e.,

which, in turn, delivers

$$
\left\|u+f_{o}\right\|_{C^{1, \alpha}(\bar{\Omega})} \leq C_{3},
$$

$$
\|J\|_{C^{\alpha}(\bar{\Omega})} \leq C_{4}
$$

for positive constants $C_{3}, C_{4}$. In particular,

$$
J(x) \geq J(y)-C_{4}|x-y|^{\alpha},
$$

for every pair of points $x, y \in \bar{\Omega}$. Now $J \equiv \operatorname{det} D f_{o}$ on $\partial \Omega$, due to $u \in W_{o}^{2, p}(\Omega)$. Thus, by (3.1) we have

$$
J(y) \geq \bar{c} \text { for all } y \in \partial \Omega .
$$

Combining this with (3.16), we conclude that there is a positive constant $\delta:=\left(\bar{c} /\left(2 C_{4}\right)\right)^{1 / \alpha}$ such that

$$
J(x) \geq \bar{c} / 2 \text { for all } x \in \bar{\Omega} \text { with } \operatorname{dist}(x ; \partial \Omega) \leq \delta .
$$

On the other hand, from (3.2) we find

$$
\int_{\Omega}(J(x))^{-q} \mathrm{~d} x \leq C E(u) \leq C \kappa=: L,
$$

where $q \geq \alpha / n$, in view of (3.3). Thus, Lemma 3.5 is applicable, i.e., there is a positive constant $\widetilde{c}$ such that

$$
J(x) \geq \widetilde{c} \quad \forall x \in \bar{\Omega} \text { with } \operatorname{dist}(x ; \partial \Omega) \geq \delta .
$$

The assertion now follows from inequalities (3.17), (3.19) and choosing $K:=\min \{\bar{c} / 2, \widetilde{c}\}$.

\section{Other boundary value PRoblems}

The proof of Theorem 3.1 ostensibly depends upon the imposition of "strong" Dirichlet conditions, i.e., $u \in W_{o}^{2, p}(\Omega), c f$. (2.5). Only these yield sufficient control close to the boundary for the proof to go through. The qualifier "strong" here refers to the fact that for smooth $u, u \equiv 0$ and $D u \equiv 0$ are imposed on sufficiently smooth portions of the boundary. In any case, a virtue of Theorem 3.1 is that no regularity of the boundary is required. For more general, physically realistic boundary conditions, we need a version of Lemma 3.5 "up to the boundary", for which mild assumptions on the latter are needed. In particular, we impose the cone condition in the sense of [1], which we now recall: given an angle $\gamma \in(0, \pi / 2]$ and a radius $\delta>0$, we let $V_{e}=V_{e}(\gamma, \delta)$ denote the open cone "in direction" $e \in S^{n-1}$ given by

$$
V_{e}:=\left\{y \in \mathbb{R}^{n}: 0<|y|<\delta, e \cdot y>\cos (\gamma)|y|\right\} .
$$

We let " $x+V_{e}$ " denote the translated cone with vertex at the point $x$. We say that $\Omega$ has the cone property if there exist an angle $\gamma$ and a radius $\delta$ such that for every $x \in \Omega$,

$$
x+V_{e(x)} \subset \Omega,
$$

for a suitable $e(x) \in S^{n-1}$.

Lemma 4.1. Let $J \in C^{\alpha}(\bar{\Omega})$ satisfy precisely the same conditions as those of Lemma 3.5, where $\Omega$ is a bounded domain that has the cone property. Then there is a positive constant $c$ such that

$$
J(y) \geq c \quad \forall y \in \bar{\Omega}
$$

where $c$ depends upon $\gamma, \delta, M, L, q$ and $n$ but not on $y, J$ or $\Omega$. 
Proof. The proof is similar to that of Lemma 3.5. For a unit vector $e \in S^{n-1}$, we now define $g:[0, \infty) \rightarrow$ $(0, \infty) \cup\{+\infty\}$ via

$$
g(t):=\int_{V_{e}}\left(t+M|x|^{\alpha}\right)^{-q} \mathrm{~d} x=\frac{\left|V_{e}\right|}{\left|B_{\delta}(0)\right|} \int_{B_{\delta}(0)}\left(t+M|x|^{\alpha}\right)^{-q} \mathrm{~d} x,
$$

where $V_{e}$ is the cone defined in (4.1). Observe that $g(t)$ is independent of $e$. As in the proof of Lemma 3.5, using $q>n / \alpha$, we note that there is a number $c>0$ such that

$$
g(t) \leq L \text { if and only if } t \geq c .
$$

Now fix $y \in \Omega$. Due to the cone property, there is unit vector $e(y)$ such that $y+V_{e(y)} \subset \Omega \cap B_{\delta}(y)$, and thus from (3.16) and (4.4) we find

$$
g(J(y)) \leq \int_{\Omega \cap B_{\delta}(y)}\left(J(y)+M|x-y|^{\alpha}\right)^{-q} \mathrm{~d} x \leq \int_{\Omega}(J(x))^{-q} \mathrm{~d} x \leq L .
$$

For $y \in \Omega$, the result follows directly from (4.5) and (4.6). Since $c$ is independent of $y$, it extends to all $y \in \bar{\Omega}$ by continuity.

We now indicate the validity of our results for other representative boundary value problems. In what follows we always assume that the bounded domain $\Omega$ has the cone property and that the boundary $\partial \Omega$ is locally Lipschitz. In particular, the latter is only needed for the embedding $W^{2, p}(\Omega) \hookrightarrow C^{1, \alpha}(\bar{\Omega}), c f$. [1].

\subsection{The weak Dirichlet problem}

We adopt all of the hypotheses of Section 2, viz., (2.2), (2.3), (2.6) and (2.7). In this case, we consider minimizing (2.4) over the admissible class

$$
\mathcal{A}_{d}:=\left\{u \in W^{2, p}(\Omega): u=0 \text { a.e. on } \partial \Omega, \operatorname{det}\left(D u+D f_{o}\right)>0 \text { a.e. in } \Omega\right\} .
$$

An obvious adjustment to the arguments in the proof of Proposition 2.1 yields the existence of a minimizer, again denoted $u_{*} \in \mathcal{A}_{d}$. Next we adopt hypothesis (3.2), (3.3) in which case we are in the situation of Lemma 4.1. Indeed, as in the proof of Theorem 3.1 with definition (3.14), we see that (3.15) and (3.18) are again valid. Hence Lemma 4.1 yields a positive constant $K$ such that

$$
\operatorname{det}\left(D u_{*}+D f_{o}\right) \geq K \text { on } \bar{\Omega},
$$

where $K$ generally depends on inf $E, f_{o}, n, p, q, \gamma, \delta$, the constants in (2.6), (3.2), and the measure of $\Omega$, but not on $u_{*}$. Again with the hypotheses of Corollary 3.3, we find that $u_{*}$ corresponds to a weak solution, i.e., (3.7) holds now for all test functions $\phi \in\left\{\zeta \in W^{2, p}(\Omega): \zeta=0\right.$. a.e. on $\left.\partial \Omega\right\}$. Finally, assuming (3.8), we again conclude

$$
u_{*}+f_{o} \text { is injective on } \bar{\Omega} \text {. }
$$

\subsection{A mixed boundary value problem}

Next we consider the typical "mixed" problem of nonlinear elasticity, whereby the placement is prescribed over only a portion of the boundary. Let $\Gamma_{o} \cup \Gamma_{1}=\partial \Omega$ denote a disjoint union such that the area measure of each subset is non-zero. In this case we minimize (2.4) over the admissible class

$$
\mathcal{A}_{m}:=\left\{u \in W^{2, p}(\Omega): u=0 \text { a.e. on } \Gamma_{o}, \operatorname{det}\left(D u+D f_{o}\right)>0 \text { a.e. in } \Omega\right\} .
$$


Proceeding as above, we obtain the existence of a minimizer $u_{*} \in \mathcal{A}_{m}$ satisfying (4.8). Again with the hypotheses of Corollary 3.3, we find that (3.7) holds now for all test functions $\phi \in\left\{\zeta \in W^{2, p}(\Omega): \zeta=0\right.$ a.e. on $\left.\Gamma_{o}\right\}$. The lack of control on $\left.\left(u_{*}+f_{o}\right)\right|_{\Gamma_{1}}$ apparently precludes (4.9).

\subsection{Further generalizations}

There are many other possibilities for mixed problems in our second-gradient theory (cf. [11]), for which Lemma 4.1 is applicable. Also, the presence of "dead load" body forces and/or surface tractions (and/or "hyper" body forces and/or "hyper" surface tractions, also now possible in our second-gradient theory) presents no difficulties to our analysis, since their inclusion simply leads to the addition of continuous linear functionals in the potential energy. For example, in the weak Dirichlet problem, let $\varpi \in L^{1}(\partial \Omega)$ be a prescribed hyper surface traction. Then the total potential energy is now (2.4) minus the linear functional

$$
L(u):=\int_{\partial \Omega} \varpi \cdot(D u \cdot n) \mathrm{d} x .
$$

where " $D u \cdot n$ " denotes the directional derivative along the unit normal $n$. Observe that $D u$ is Hölder continuous (Rem. 2.2), while the unit normal vector field $n$ exists a.e. on $\partial \Omega$, cf. [12]. Clearly $L$ is bounded on $W^{2, p}(\Omega) \hookrightarrow$ $C^{1, \alpha}(\bar{\Omega})$.

\section{Concluding Remarks}

The additive, second-gradient energy density $\Psi(\cdot)$ in (2.4), characterized by convexity, represents one of the simplest, physically realistic models within the category of second-gradient elasticity. The Cahn-Hilliard model is of this type, viz., $\Psi(G)=\frac{\epsilon}{2}|G|^{2}$, where $\epsilon>0$ is a small parameter. Note that our results are not applicable to that case, however, due to the required growth conditions, $c f$. (2.3), (2.6). Proposition 2.1 holds under the weaker assumption that $\Psi(\cdot)$ be quasiconvex [8-10].

Our results are also applicable to sufficiently smooth deformations with finite energy in classical nonlinear elasticity ( $\Psi \equiv 0$ in (2.4)). Recall that if in addition to $(2.7), \Phi(\cdot)$ is polyconvex and satisfies appropriate growth conditions, then the existence of a minimizer in $W^{1, p}(\Omega)$ is well known [2]. Hence, in particular, if it happens that such a minimizer $u_{*}$ also belongs to $C^{1, \alpha}(\bar{\Omega})$, then Corollaries 3.2 and 3.3 hold, as well as Corollary 3.4 for $u=u_{*}$. In this regard, our results overlap with those [6], which treats sufficiently smooth minimizers in classical nonlinear elasticity. In particular, with the presumption that a minimizer is in $C^{1, \alpha}(\bar{\Omega})$, the equivalent of Corollary 3.2 is established in [6]. Moreover, the proof of Theorem 2.5 in [6] relies on a nonconstructive argument closely related to Lemma 3.5. In contrast to our approach, it exploits that (in our notation) $\left(\operatorname{det}\left(D u_{*}+D f_{0}\right)\right)^{-s}$ is integrable for any $s>0$. In our case, the latter is guaranteed only for $s \leq q$, directly by finite energy alone, whereas in [6] it is observed that this integrability can be obtained (for arbitrary $s>0$ ), exploiting the fact that a minimizer $u_{*}$ is always a weak solution of the Eshelby conservation law [3,4]. However, this requires $\Phi$ to be of special form (cf. (2.1)-(2.3) in [6]), while we, on the other hand, have to assume (2.3) and (3.3). Our method thus provides an alternative approach - constructively from Lemma 3.5 or Lemma 4.1 and without direct appeal to the Eshelby conservation law. Finally we emphasize that the arguments in [6], leading to the positivity of the determinant of the deformation, genuinely require energy minimality (via the Eshelby conservation law), whereas our approach holds for all admissible deformations of bounded energy.

Also note that for the purposes of the present paper, avoiding the generalized version of the latter is essential. That is, any attempt to use it in an analogous way as in [6] apparently fails unless one is willing to impose severe additional regularity assumptions on the solution to deal with the terms associated with the second gradient term in the energy.

Acknowledgements. The work of T.J.H. was supported in part by the National Science Foundation through grant DMS0707715, which is gratefully acknowledged. 


\section{REFERENCES}

[1] R.A. Adams, Sobolev Spaces. Academic Press, New York (1975).

[2] J.M. Ball, Convexity conditions and existence theorems in nonlinear elasticity. Arch. Rational Mech. Anal. 63 (1977) $337-403$.

[3] J.M. Ball, Minimizers and Euler-Lagrange Equations, in Proceedings of I.S.I.M.M. Conf. Paris, Springer-Verlag (1983).

[4] J.M. Ball, Some open problems in elasticity, in Geometry, Mechanics and Dynamics, P. Newton, P. Holmes and A. Weinstein Eds., Springer-Verlag (2002) 3-59.

[5] P. Bauman, N.C. Owen and D. Phillips, Maximum principles and a priori estimates for a class of problems from nonlinear elasticity. Ann. Inst. H. Poincaré Anal. Non Linéaire 8 (1991) 119-157.

[6] P. Bauman, D. Phillips and N.C. Owen, Maximal smoothness of solutions to certain Euler-Lagrange equations from nonlinear elasticity. Proc. Royal Soc. Edinburgh 119A (1991) 241-263.

[7] P.G. Ciarlet, Mathematical Elasticity Volume I: Three-Dimensional Elasticity. Elsevier Science Publishers, Amsterdam (1988).

[8] B. Dacorogna, Direct Methods in the Calculus of Variations. Springer-Verlag, New York (1989).

[9] G. Dal Maso, I. Fonseca, G. Leoni and M. Morini, Higher-order quasiconvexity reduces to quasiconvexity. Arch. Rational Mech. Anal. 171 (2004) 55-81.

[10] E. Giusti, Direct Methods in the Calculus of Variations. World Scientific, New Jersey (2003).

[11] E.L. Montes-Pizarro and P.V. Negron-Marrero, Local bifurcation analysis of a second gradient model for deformations of a rectangular slab. J. Elasticity 86 (2007) 173-204.

[12] J. Nečas, Les Méthodes Directes en Théorie des Équations Elliptiques. Masson, Paris (1967).

[13] X. Yan, Maximal smoothness for solutions to equilibrium equations in 2D nonlinear elasticity. Proc. Amer. Math. Soc. 135 (2007) 1717-1724. 